\title{
Doppler echocardiographic assessment of subclinical valvitis in the diagnosis of acute rheumatic fever
}

\author{
Cleonice C. Mota \\ Universidade Federal de Minas Gerais - Faculdade de Medicina, Serviço de Cardiologia e Cirurgia Cardiovascular, Belo \\ Horizonte, Brazil
}

A $T$ THE BEGINNING OF A NEW CENTURY, almost 200 years after the initial descriptions of the cardiac involvement by Wells in 1812, and by Jean-Baptiste Bouillaud in 1836, rheumatic fever remains a significant challenge to those involved in providing health care. ${ }^{1}$ Although impressive data have been gathered by studies in several areas of investigation, all this knowledge has been unable to explain the pathogenesis of the condition. In many parts of the world, the origin of relevant problems in the cardiovascular area is still related to rheumatic fever, with significant rates of morbidity and mortality. In developing countries, the disease is a risk because of its cardiac sequels, being the most important cause of acquired heart disease in the young. Its potential resurgence now emphasises its burgeoning significance in the developed world.

Several factors, nonetheless, have contributed to a changing pattern of rheumatic fever. The decline in frequency and seriousness during the last decades has been attributed to changes in the host and environment, besides possible modifications in virulence of the streptococcus. Other factors influencing the changes include the increased ability to diagnose the disease through the establishment of stricter diagnostic criterions, as well as the strategies of primary and secondary prophylaxis for controlling recurrences. Furthermore, procedures to assess damage to the heart, and to improve the cardiac performance, have resulted in more effective health care by the introduction of technological advances, mainly Doppler echocardiog-

Correspondence to: Dra Cleonice de C Mota, Universidade Federal de Minas Gerais - Faculdade de Medicina, Serviço de Cardiologia e Cirurgia Cardiovascular, Rua Cabralia 30/501, Belo Horizonte, Minas Gerais 30240-080, Brazil. Tel: 0055 239 7198; Fax: 005531282 5357; E-mail: cleomota@ medicina.ufmg.br raphy, valvoplasty, and surgical valvar replacement and repair.

\section{The clinical approach to cardiac involvement}

The inadequate understanding of pathophysiological mechanisms, along with the absence of pathognomonic clinical features and specific laboratory tests, have produced difficulties in achieving the correct diagnosis of rheumatic fever. Furthermore, this disease, involving multiple systems of organs, presents a large spectrum of symptoms and signs, usually appearing in several combinations, and greatly varying in severity. Since many conditions have similar manifestations, the differential diagnosis is frequently difficult, and is mostly related to isolated presentation of arthritis or carditis.

With the intention of establishing a systematic guide for diagnosis, Jones introduced, in 1944, a set of clinical and laboratorial data, systematised by means of categories. ${ }^{2}$ The distinction between major and minor criterions was mostly based on the specificity for diagnosis, rather than on frequency of occurrence. Over the years, these guidelines have undergone periodical review. Over four revisions, the modifications introduced have enhanced specificity, and have also provided more diagnostic information by presenting new requirements and more detailed description of manifestations. But, despite these advances, and even subsequent to (almost) more than fifty years of progress after the first publication of the Jones criterions, few contributions derived from the new technologies have served to improve the diagnosis of rheumatic fever. ${ }^{3}$

Carditis is, potentially, the most severe manifestation of the disease, because it is the only one which results in significant sequels and death. Apart from emphasising the importance of 
prophylaxis, the potential for permanent damage to the heart reinforces the need for an accurate and early diagnosis. Marked clinical evidence of cardiac involvement, particularly if associated with other major manifestations of the Jones criterions, results in a more characteristic presentation of the disease. On the other hand, the disease may present a more challenging picture when carditis is mild.

During the acute phase, the inflammatory process can involve all three components of the heart. The valves are involved most typically, in terms of both frequency and severity. Pericarditis is probably the least common clinical finding and, as with myocarditis, should not be considered rheumatic in origin in the absence of valvar involvement. As is well known, due to the much higher frequency of left-sided valvar lesions, the diagnosis of rheumatic valvitis should always be called into question in the setting of exclusively right-sided presentation. Mild or greater degrees of tricuspid and pulmonary regurgitation may be found in subjects with normal hearts as a result of volume overload or pulmonary hypertension. ${ }^{4}$ Since the clinical findings of carditis are mostly represented by the auscultatory findings of valvitis, and the magnitude seems to be determined mostly by the severity of the valvar lesions, it is exceedingly difficult to identify minor degrees of abnormalities using clinical methods. If found with no clinical findings, this should be considered as subclinical carditis.

Pathological valvar regurgitation of a mild degree, detected only by Doppler echocardiography, nonetheless, has also been identified during the chronic phase in patients with previously unsuspected valvar disease by physical examination, this occurring without clinical evidence of carditis during the acute phase. This picture characterises a chronic subclinical valvar disease. ${ }^{5}$

In areas with a high prevalence of rheumatic fever, furthermore, it is by no means rare to find that cardiac involvement becomes evident only in the chronic phase, being manifest through valvar sequels with their obvious clinical manifestations. First, in terms of overall evolution, the presence of subclinical carditis might have implications in the development of chronic rheumatic heart disease among patients with no clinical signs of cardiac involvement during the acute phase. Second, the more representative symptoms and signs related to arthritis and chorea might then represent a stronger reason to seek medical assistance than solitary and vague symptoms of cardiac involvement. Additionally, mild clinical manifestations identified during the physical examination could be misinterpreted as being due to the hyperdynamic state of the acute phase. The valvar lesions could then be made worse by recurrent attacks of carditis. Considering the mimetic propensity of the clinical manifestations, the risk of a recurrent and more severe involvement of the heart during subsequent acute episodes is increased. At the moment, except for evidence of recurrence, information is lacking about the other factors which could possibly contribute to the evolution of valvar damage.

\section{Subclinical valvitis and its echocardiographic assessment}

Until the advent of Doppler echocardiography, the diagnosis of valvitis had been based exclusively on the presence of auscultatory findings and radiographic and electrocardiographic abnormalities. Although these tests are of considerable clinical benefit, they are characterised by low specificity and low sensitivity, apart from the fact that normal findings do not exclude minor degrees of cardiac involvement.

Doppler echocardiography, therefore, has become an important tool when evaluating cardiac involvement through systematic investigation of morphological and functional aspects of the heart. It has been recognised being particularly useful in identifying lesions and assessing the degree of cardiac involvement in rheumatic fever, providing valuable information for the subsequent clinical management.

During the acute phase of rheumatic fever, it is a fact that minor degrees of pathological regurgitation can be present in the absence of positive clinical signs, just as it is also a fact that the echocardiographic study, more sensitive than auscultation, is a powerful method of detecting the minor degree of lesions seen in the setting of subclinical valvitis. It is essential, nonetheless, that strict Doppler echocardiographic criterions be established to differentiate pathological from physiological regurgitation so as to avoid overdiagnosis, since valvar leaks are frequently observed in subjects with structurally normal hearts.

In 1992, the committee establishing the latest revision of the Jones Criterions concluded that the available data at that time was not enough to include echocardiographic findings without corresponding auscultatory abnormalities as a criterion for the diagnosis of valvitis in rheumatic fever. ${ }^{6}$ In spite of the difficulties in making comparative analysis due to different methodological approaches, initial reports have given valuable information about the pattern and frequency of physiological regurgitation, and have provided data to confirm the existence of valvitis in the 
absence of clinical signs in patients having rheumatic fever. ${ }^{7-12}$ More recently, additional investigations and discussions have implemented these experiences. Stricter Doppler echocardiographic criterions have now been established, therefore, to permit a more confident differentiation between the characteristics of physiological and pathological valvar regurgitation. ${ }^{4,13-16}$

It remains a fact, however, that the lack of an accurate diagnosis of cardiac lesions can contribute to the underdiagnosis and, consequently, interfere in the management of patients. The use of anti-inflammatory medication is required for carditis, but is not required for pure chorea. Furthermore, extended bed rest is required for patients with known cardiac involvement. Besides the necessity of additional prophylaxis for infective endocarditis, the duration of secondary prophylaxis in the presence of valvitis is longer than that used for isolated arthritis and/or chorea. In addition, echocardiographic diagnosis of the subclinical valvitis can also represent additional data, enhancing the chance of a correct diagnosis of rheumatic fever in these patients with isolated arthritis and/or chorea.

On the other hand, the echocardiographic diagnosis of valvitis should be considered as a finding existing within a wider diagnostic context. It is necessary to bear in mind that the echocardiographic findings do not confirm the aetiology of the disease, and do not constitute a substitute for complete clinical investigation. The echocardiographic evaluation must be seen as a highly accurate and sensitive technique, which, if adequately incorporated, will improve the diagnosis by providing earlier evidence of valvar involvement. Uncertainty, however, will continue to exist. In such instances, a period of individual observation should be required in order to assess the evolutionary data before arriving at a firm conclusion. Although complementary laboratory data should always be used to achieve a more precise evaluation, diagnostic decisions are oriented by clinical judgement.

As part of the Program of Study and Control of Rheumatic Fever ${ }^{17,18}$ established at the Federal University of Minas Gerais, we carried out two studies within the Division of Paediatric Cardiology to investigate subclinical valvitis. Using a cohort of patients with a follow-up ranging from 2 to 15 years, and resulting in 1383 patient years, we evaluated the diagnostic aspects, evolution, and prognosis of 258 children and adolescents with rheumatic fever. During the acute phase, $87.2 \%$ of 109 patients were found to have cardiac involvement by Doppler echocardiography, $15.6 \%$ of these having mild valvitis in the absence of clinical signs. In those with moderate and severe valvar lesions, there was concordance between the clinical evaluation and the Doppler echocardiographic findings. ${ }^{17}$ In the second study, which involved blind Doppler echocardiographic investigation of rheumatic and healthy children, we found subclinical valvitis in $23.1 \%$ of patients with clinical manifestations represented by isolated arthritis or chorea. We also confirmed the pattern of regurgitant jets as previously described in normal subjects (Lanna and colleagues, unpublished data). During the follow-up, which still continues, it seems that, in the absence of recurrences, there is no tendency for the valvar lesions to get worse.

\section{Conclusion}

When using strict criterions, therefore, it is justified to argue that subclinical valvitis diagnosed by Doppler echocardiography should be considered as mild carditis, thus becoming a major criterion in the diagnosis of acute rheumatic fever. Yet major questions remain. Considering that the prognosis of rheumatic fever is closely related to the involvement of the heart and the risks of recurrences, what will be the impact of identification of subclinical carditis in terms of the late outcome? Has subclinical valvitis the same potential for evolution as those cardiac lesions which are identified by clinical examination? Considering the implications for use of additional resources subsequent to the inclusion of Doppler echocardiographic criterions in the routine laboratory investigation of rheumatic fever, will the costs be greater than the economic and social benefits achieved in terms of avoidance of potential sequels? Taking all these circumstances into account, the data now available suggests that although we must remain cautious, we must give the same importance to valvitis diagnosed by Doppler echocardiography as to that seen clinically. It is essential, therefore, that we undertake further rapid investigations to corroborate this approach. ${ }^{19}$

\section{References}

1. Acierno LJ. The history of cardiology. Parthenon Publishing, London, 1994.

2. Jones TD. Diagnosis of rheumatic fever. JAMA 1944;127: $481-484$

3. Shaiffman RN. Guidelines maintenance and revision, 50 years of the Jones criteria for diagnosis of rheumatic fever. Arch Pediatr Adolescent Med 1995; 149: 727-732.

4. Wilson NJ. \& Neutze JM. Editorial: Echocardiographic diagnosis of subclinical carditis in acute rheumatic fever. Intern J. Cardiol 1995; 50: 1-6. 
5. Meira ZMA. Cardiopatia reumática em crianças e adolescentes: aspectos clínicos e ecoDopplercardiogárficos, evolução e prognótico (Rheumatic heart disease in children and adolescents: clinical and Doppler echocardiographic aspects, evolution and prognostic factors), Dec. 2000; Belo Horizonte, Brazil, Federal University of Minas Gerais, 207p. Medicine $\mathrm{PhD}$ Thesis.

6. Special Writing Group of the Committee of Rheumatic Fever, Endocarditis, and Kawasaki Disease of the Council on Cardiovascular Disease in the Young of the American Heart Association. Guidelines for the diagnosis of rheumatic fever. Jones criteria, 1992 update. JAMA 1992; 268: 2069-2073.

7. Brand A., Dollberg S, Keren A. The prevalence of valvular regurgitation in children with structurally normal hearts: a colour Doppler echocardiographic study. Am Heart J 1992; 123: 177-180.

8. Yoshida K, Yoshikawa J, Shakudo M, Akasaka T, Jyo Y, Takao S, Shiratori K, Koizumi K, Okumachi F, Kato H, Fukaya T. Color Doppler evaluation of valvular regurgitation in normal subjects. Circulation 1988; 78: 840-847.

9. Choong CY, Abascal VM, Weyman J, Levine RA, Gentile F, Thomas JD, Weyman AE Prevalence of valvular regurgitation by Doppler echocardiography in patients with structurally normal hearts by two-dimensional echocardiography Am Heart J 1989; 117: 636-642.

10. Steinfeld L, Ritter S, Rappaport H, MartinezE. Silent rheumatic mitral regurgitation unmasked by Doppler studies (abstracts). Circulation 1986; 74 Suppl 11: 385.

11. Veasy LG, Wiedmeier SE, Orsmond GS, Ruttenberg HD, Boucel MM, Roth SJ, Tait VF, Thompson Jòb, Daly Jòb,
Kaplan EL, Hill HR. Resurgence of acute rheumatic fever in the intermountain area of the United States. N Engl J Med 1987;316:421-427.

12. Folger GM, Hajar R. Doppler echocardiographic findings of mitral and aortic valvular regurgitation in children manifesting only rheumatic arthrit is. Am J. Cardiol 1989; 63: 1278-1280.

13. Folger Jr. GM, Hajar R, Robida A, Hajar HA. Occurrence of valvar heart disease in acute rheumatic fever without evident carditis: colour-flow Doppler identification. Br Heart J 1992; 67: 434-438.

14. Abernethy M, Bass, N, Sharpe N, Grant C, Neutze J, Clarkson P, Greaves S, Lennon D, Snow S, Whalley G. Doppler echocardiography and the early diagnosis of carditis in acute rheumatic fever. Aust NZ J Med 1994; 24: 530-535.

15. Minich LL, Tani LY, Pagotto LT, Shaddy RE, Veasy LG. Doppler echocardiography distinguishes between physiologic and pathologic "silent" mitral regurgitation in patients with rheumatic fever. Clin Cardiol 1997; 20: 924-926.

16. Narula J, Chandrasekhar Y, Rahimtoola S. Diagnosis of active rheumatic carditis. The echoes of change. 1999; 100: 1576-1581.

17. Mota CCC, Meira ZMA, Graciano RN, Silva MC. Diagnostic aspects of streptococcal infections carditis and other acute forms. Cardiol Young 1992; 2: 222-228.

18. Mota CCC, Meira ZMA . Rheumatic fever. Cardiol Young 1999; 8: 98-109.

19. Özkutlu S, Ayabakan C, Saraçlar M. Can subclinical valvitis detected by echocardiography be accepted as evidence of carditis in the diagnosis of acute rheumatic fever? Cardiol Young 2001; 11: 255-260 